\title{
Sinonasal Inverted Papilloma Associated with Adenoid Cystic Carcinoma
}

\author{
Yohei Takada ${ }^{a}$ Mikiya Asako ${ }^{a}$ Risaki Kawachi ${ }^{b}$ Tomoko Takadac \\ Hiroshi Iwai ${ }^{b}$ \\ aDepartment of Otolaryngology - Head and Neck Surgery, Kansai Medical University \\ Hospital Medical Center, Osaka, Japan; ${ }^{b}$ Department of Otolaryngology - Head and Neck \\ Surgery, Kansai Medical University Hospital, Osaka, Japan; 'Department of Otolaryngology, \\ JCHO Hoshigaoka Medical Center Hospital, Osaka, Japan
}

\author{
Keywords \\ Inverted papilloma · Adenoid cystic carcinoma $\cdot$ Malignant transformation · Synchronous \\ carcinoma
}

\begin{abstract}
Inverted papilloma is the most common benign tumor of the paranasal sinuses with the possibility of malignant transformation. On the one hand, adenoid cystic carcinoma (ACC) is a rare malignant neoplasm that arises from the secretory glands. Sinonasal ACC accounts for $10 \%-$ $25 \%$ of all head and neck ACC. This neoplasm is defined by its distinctive histologic appearance. Surgical resection, whenever possible, is the mainstay therapy. An association between inverted papilloma and malignancy is controversial. A synchronous carcinoma has been established at diagnosis in $3.3 \%-11 \%$ of cases, and the risk of metachronous carcinoma is $<3 \%$. We report a case of an 84 -year-old female affected with sinonasal inverted papilloma associated with synchronous ACC. She had right nasal obstruction due to the rapid growth of the tumor. She was referred to our department for further workup. A frozen biopsy revealed part of the tumor as ACC. The tumor was consistent with preoperative imaging, and surgical findings also showed infiltration into the orbit. There was no consent for radical surgery with orbital exenteration and adjuvant chemoradiotherapy in consideration of her advanced age. This is the first case report of the association between sinonasal inverted papilloma and ACC within our retrieval capability.
\end{abstract}

\section{Karger"}




\section{Introduction}

Although inverted papilloma is the most common benign tumor of the paranasal sinuses, it has high local recurrence. It may also cause malignant transformation or complications with malignant tumors such as squamous cell carcinoma (SCC) [1-3].

On the other hand, adenoid cystic carcinoma (ACC) is a rare malignant neoplasm that arises from the secretory glands. It affects the major and minor salivary glands of the head and neck. It is the second most common tumor of the nasal cavity and paranasal sinuses. Sinonasal ACC accounts for $10 \%-25 \%$ of all head and neck ACC.

A synchronous carcinoma has been established at diagnosis in $3.3 \%-11 \%$ of cases, and the risk of metachronous carcinoma has been shown to be $<3 \%$. This is a case report of inverted papilloma associated with ACC. The findings are discussed with the existing medical literature.

\section{Case Report}

An 84-year-old female with a polyp in the right nose was referred to our institution from another hospital. Pertinent past medical history included hypertension and tuberculosis. She was a nonsmoker and nonalcoholic beverage drinker. There was a rapid increase of the mass within 1 month, and biopsy was done. The biopsy result showed inverted papilloma. She was suspected of having a malignant tumor.

Anterior rhinoscopy showed a mass lesion extending to the anterior nares in the right nostril (Fig. 1a). Visualization of the posterior nasal cavity was difficult due to the obstruction of the mass (Fig. 1b). There were no eye symptoms such as diplopia or ocular motility disorder. CT imaging showed that the soft tissue concentration shadow that filled the nasal cavity extended from the nostrils to the maxillary sinus and nasopharynx, and that some of lamella of orbital of ethmoid bone may have osteolytic changes secondary to the malignancy (Fig. 1c, d). MRI was performed to evaluate the shape and extension of the mass. T2-weighted axial and coronal MRI showed a mixture of high and low signals (Fig. 1e, f). The mass was a stage equivalent to T4 using the inverted papilloma staging system proposed by Krouse [4]. Positron emission tomography (PET) examination showed no suspected metastasis. Preoperatively, inverted papilloma was suspected based on the neoplastic lesion filling the right nasal cavity and the results on CT/MRI. A complication of SCC based on the rate of tumor growth was suspected.

Surgery with biopsy was scheduled under general anesthesia to obtain a definitive diagnosis. Piecemeal resection of the tumor protruding from the external right nostril was performed first.

The area was packed by cotton with $4 \%$ lidocaine and epinephrine. Then, volume reduction of the inferior turbinate and tumor was done. The agger nasi was injected with $2 \mathrm{~mL}$ of $0.5 \%$ lidocaine with epinephrine solution to reduce the bleeding.

A polyp was found in the area around the tumor. The polyp and inverted papilloma were mixed and resected using a microdebrider. The base of the tumor was suspected to be on the posterior wall of the maxillary sinus extending inside the orbit; it had a hard part with different properties of inverted papilloma (Fig. 2a). A part of the tumor was resected and submitted for frozen biopsy, and ACC was confirmed. During dissection, the tumor adhered to the orbital lamina of the ethmoid bone (Fig. 2b). We explained to the patient's family intraoperatively that the tumor adhered to the orbit, with the risk of orbital damage. It was then decided to maximize the removal of the tumor without orbital damage. Some tumor remained in the orbital lamina of the ethmoid bone upon termination of the operation.

On hematoxylin and eosin staining, stratified squamous epithelium with a papillomalike structure showing an enlarged introverted growth was observed; these were distinct

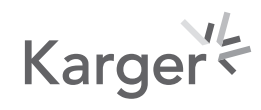




\section{Case Reports in Oncology}
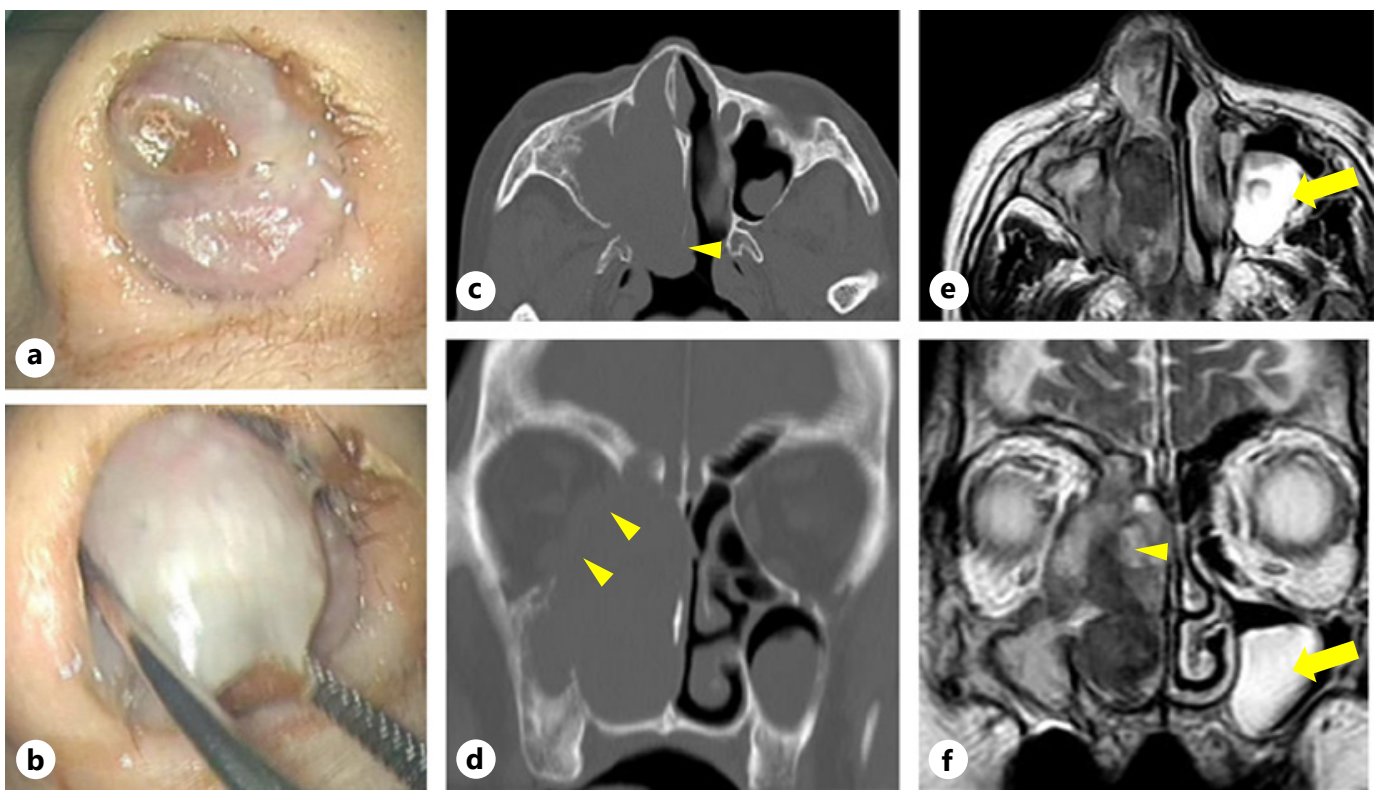

Fig. 1. a A mass extending to the anterior nares in the right nostril. $\mathbf{b}$ Observation of the back of the nasal cavity was difficult because it was full. c Axial CT shows that the soft tissue density lesion in the right nasal cavity and maxillary sinus extends to the posterior nostrils (arrowhead). d Coronal CT shows an invasive soft tissue mass filling the right nasal cavity, extending into the right orbit (arrowhead). T2-weighted axial (e) and coronal (f) MRI shows a mixture of high and low signals in the right nasal cavity. Polyp findings in the left maxillary sinus (arrow).
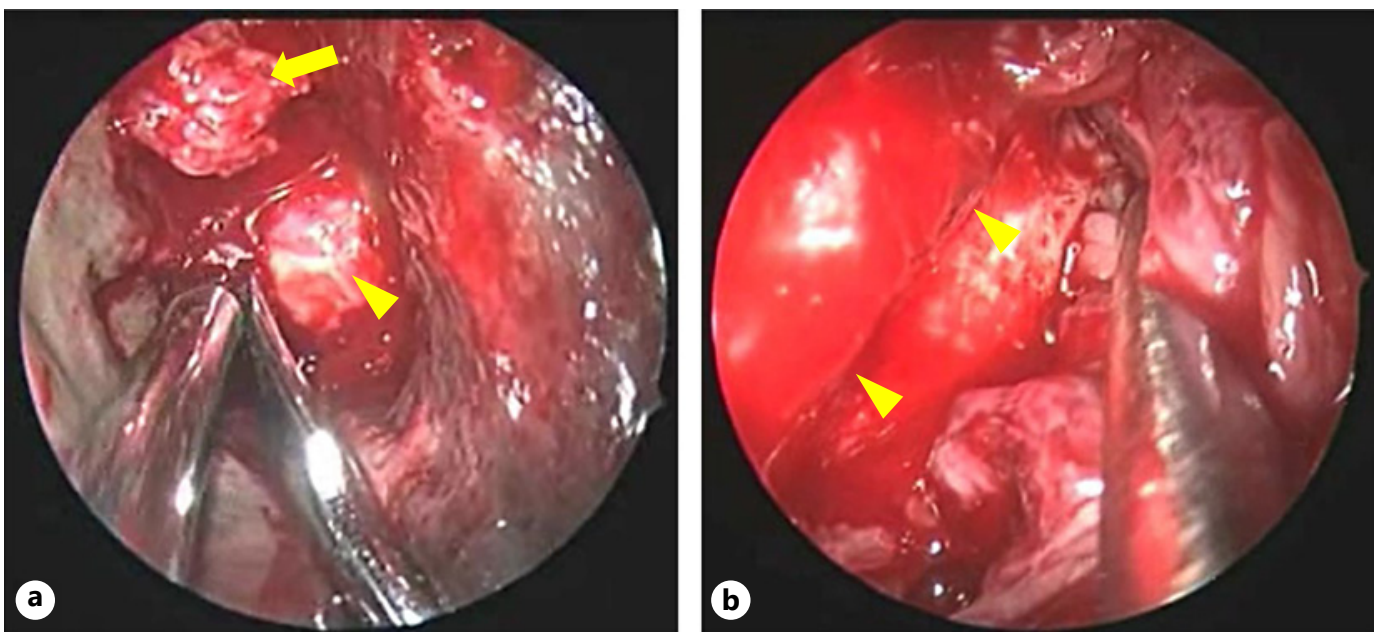

Fig. 2. a A hard tumor was confirmed by excising the polyp and inverted papilloma (arrow) around the tumor (arrowhead). b Tumor adheres to the lamella of the orbit.

characteristics of inverted papilloma (Fig. 3a). The tumor was associated with ACC in the minor salivary glands. Some components of the ACC were found on the superficial layer (Fig. 3b). The excision margin was positive. There was a cribriform pattern forming a sieve-like alveolar portion including a small cyst-like cavity. It was grade I in Szanto et al. [5] classification.

The main consideration during preoperative discussions was the patient's advanced age. Hence, invasive extended surgery such as combined resection in the orbit was not desired. 


\section{Case Reports in Oncology}
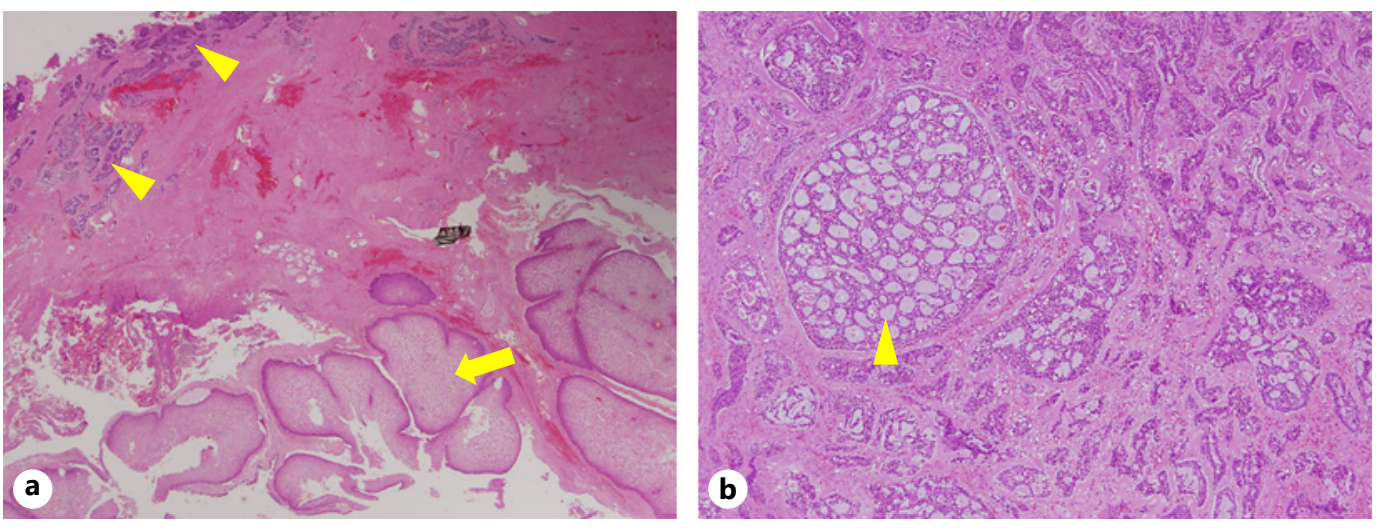

Fig. 3. Postoperative pathology. a H/E staining $(\times 2)$ shows a clear border between inverted papilloma (arrow) and ACC (arrowhead). This indicates that the ACC was combined with inverted papilloma. In some parts, the components of ACC were exposed on the superficial layer. b H/E staining $(\times 4)$ shows the cribriform pattern of ACC (arrowhead). ACC, adenoid cystic carcinoma.

As expected prior to the surgery, the tumor was partially malignant as seen on frozen biopsy, and intraorbital invasion was observed. The operation was completed with some tumor remnants left in situ; this decision was made upon consultation with the patient's family. Postoperative radiotherapy was not desired, and hence it was decided to monitor the patient's progress with the malignancy. She showed gradual growth in the tumor, but died of cerebral hemorrhage 5 years after surgery without any apparent metastasis.

\section{Discussion}

To the best our knowledge, this is the first case of sinonasal inverted papilloma associated with ACC. The nasal sinus region includes skin, mucous gland, glandular tissue, vascular nerves, cartilage, and bone, among others. Various tumors originate from these structures, hence the different histopathological findings. Most are benign tumors, with the most common finding of nasal sinus papilloma. It is classified into 3 histological types: outgoing, inverted, and columnar epithelial.

Inverted papilloma has the tendency for local recurrence, malignant transformation, and complication of malignant tumors such as SCC. Majority of malignant cases are SCC that can be synchronous or metachronous with inverted papilloma. The rate of synchronous growth of a malignancy and inverted papilloma is at 3.3\%-11\%. On the one hand, the rate of metachronous growth is at $0.3 \%-3 \%$ [1-3]. Therefore, in cases where inverted papilloma is suspected, it is necessary to assume complications and transformation of malignant tumors.

ACC is a rare malignant tumor that usually arises in the major and minor salivary glands $[5,6]$. It accounts for about $1 \%$ of all head and neck malignancies and about $6 \%-10 \%$ of malignant salivary glands [7]. It is the second most common tumor of the sinonasal area. Sinonasal ACC accounts for $10 \%-25 \%$ of all head and neck ACC [8].

ACC proliferates slowly and has a good short-term prognosis. However, it can infiltrate stroma, especially around the nerve, and has a high rate of local recurrence [9]. Although there are few lymph node metastases, distant metastases are relatively high. Therefore, the longterm prognosis is poor $[10,11]$. Surgical resection is the first-line treatment. The effectiveness of postoperative radiation has also been pointed out $[12,13]$, but new treatment methods that improve long-term treatment results have not yet been established.

\section{Karger's}


There are various reports of inverted papilloma associated with SCC [1-3]. There are also reports of inverted papilloma with malignant lymphoma [14], as well as recurrence with sinonasal undifferentiated carcinoma [15]. However, there are no reports of cases with ACC.

The patient in this case report had a preoperative diagnosis of inverted papilloma through biopsy. The rapid growth of the mass increased the suspicion of inverted papilloma with SCC. Another biopsy was performed under general anesthesia for definitive diagnosis. A rapid intraoperative histopathological examination gave a diagnosis of ACC instead of the expected SCC. The recommended treatment is resection of the tumor with normal tissue margins. Since the tumor was expected to infiltrate the orbit, a margin of intraorbital tissue should have been removed through an invasive approach by external incision. Adjunctive radiation and chemotherapy are often done after surgery.

However, due to the patient's advanced age, the family avoided the risk of intraorbital injury and requested surgery for diagnostic purposes instead of total tumor resection. They also did not consent for postoperative adjuvant treatment. Although the ACC had grown postoperatively, it had a cribriform pattern, and the grade was relatively low; hence, it is likely that the tumor did not directly cause her death. The patient died of cerebral hemorrhage 5 years after the operation.

\section{Conclusion}

This is the first case report of the sinonasal inverted papilloma associated with ACC. The patient was suspected of having a malignant tumor because of the rapid increase of the mass in the right nasal cavity. Resection with biopsy was performed for definitive diagnosis. Radical surgery of the orbit and adjuvant chemoradiation were not performed due to her advanced age. The tumor had grown on follow-up, but the patient died of cerebral hemorrhage 5 years after the operation.

\section{Acknowledgment}

We would like to thank Editage (www.editage.com) for English language editing.

\section{Statement of Ethics}

Written informed consent was obtained from the patient's family for the publication of this case report and any accompanying images. A copy of the written consent is available for review by the Editor-in-chief of this journal.

\section{Conflict of Interest Statement}

The authors have no conflicts of interest to declare.

\section{Funding Sources}

The authors received no financial support for the research, authorship, and publication of this article. 


\section{Case Reports in Oncology}

\begin{tabular}{l|l}
\hline Case Rep Oncol 2021;14:1429-1434 \\
\hline DOI: 10.1159/000519437 & $\begin{array}{l}\text { @ 2021 The Author(s). Published by S. Karger AG, Basel } \\
\text { www.karger.com/cro }\end{array}$ \\
\hline
\end{tabular}

Takada et al.: Sinonasal Inverted Papilloma with Adenoid Cystic Carcinoma

\section{Author Contributions}

Y.T. and T.T. performed the literature research and composed the manuscript. M.A. and K.K. performed the surgeries. H.I. critically revised the manuscript. Y.T., M.A., and K.K. performed pre- and postoperative examinations of the patient, provided photographs, and drafted a first version of the manuscript. All authors approved the final manuscript.

\section{Data Availability Statement}

All data generated or analyzed during this study are included in this article. Further enquiries can be directed to the corresponding author.

\section{References}

1 Mirza S, Bradley PJ, Acharya A, Stacey M, Jones NS. Sinonasal inverted papillomas: recurrence, and synchronous and metachronous malignancy. J Laryngol Otol. 2007;121(9):857-64.

2 Choi JW, Kim SG, Kim YM, Yoon YH, Kim AY, Rha KS. Clinical and histologic features of inverted papillomaassociated malignancy. Eur Arch Otorhinolaryngol. 2012;269(11):2349-54.

3 Kim DY, Hong SL, Lee CH, Jin HR, Kang JM, Lee BJ, et al. Inverted papilloma of the nasal cavity and paranasal sinuses: a Korean multicenter study. Laryngoscope. 2012;122(3):487-94.

4 Krouse JH. Development of a staging system for inverted papilloma. Laryngoscope. 2000;110(6):965-8.

5 Szanto PA, Luna MA, Tortoledo ME, White RA. Histologic grading of adenoid cystic carcinoma of the salivary glands. Cancer. 1984;54(6):1062-9.

6 Spiro RH, Huvos AG, Strong EW. Adenoid cystic carcinoma of salivary origin. A clinicopathologic study of 242 cases. Am J Surg. 1974;128(4):512-20.

7 Michel G, Joubert M, Delemazure AS, Espitalier F, Durand N, Malard O. Adenoid cystic carcinoma of the paranasal sinuses: retrospective series and review of the literature. Eur Ann Otorhinolaryngol Head Neck Dis. 2013;130(5):257-62.

8 Rhee CS, Won TB, Lee CH, Min YG, Sung MW, Kim KH, et al. Adenoid cystic carcinoma of the sinonasal tract: treatment results. Laryngoscope. 2006;116(6):982-6.

9 Vrielinck LJ, Ostyn F, van Damme B, van den Bogaert W, Fossion E. The significance of perineural spread in adenoid cystic carcinoma of the major and minor salivary glands. Int J Oral Maxillofac Surg. 1988;17(3):190-3.

10 Spiro RH, Huvos AG, Strong EW. Adenoid cystic carcinoma: factors influencing survival. Am J Surg. 1979; 138(4):579-83.

11 Spiro RH, Huvos AG. Stage means more than grade in adenoid cystic carcinoma. Am J Surg. 1992;164(6):623-8.

12 Vikram B, Strong EW, Shah JP, Spiro RH. Radiation therapy in adenoid-cystic carcinoma. Int J Radiat Oncol Biol Phys. 1984;10(2):221-3.

13 Takagi D, Fukuda S, Furuta Y, Yagi K, Homma A, Nagahashi T, et al. Clinical study of adenoid cystic carcinoma of the head and neck. Auris Nasus Larynx. 2001;28 Suppl:S99-102.

14 Hsu HJ, Huang CC, Chuang MT, Tien CH, Lee JS, Lee PH. Recurrent inverted papilloma coexisted with skull base lymphoma: a case report. World J Clin Cases. 2021;9(2):516-20.

15 Kim JS, Hong KH, Jang KY, Song JH. Sinonasal undifferentiated carcinoma originating from inverted papilloma: a case report. Medicine. 2017;96(45):e8584.

\section{Karger' ${ }^{\prime \prime}$}

